\title{
Dynamics in Braess Paradox with Nonimpulsive Commuters
}

\author{
Arianna Dal Forno, ${ }^{1}$ Ugo Merlone, ${ }^{2}$ and Viktor Avrutin ${ }^{3,4}$ \\ ${ }^{1}$ Department of Economics and Statistics "Cognetti de Martiis," University of Torino, 10153 Torino, Italy \\ ${ }^{2}$ Department of Psychology, University of Torino, 10124 Torino, Italy \\ ${ }^{3}$ DESP, University of Urbino "Carlo Bo," 61026 Urbino, Italy \\ ${ }^{4}$ IST, University of Stuttgart, 70550 Stuttgart, Germany
}

Correspondence should be addressed to Ugo Merlone; ugo.merlone@unito.it

Received 16 May 2014; Accepted 27 July 2014

Academic Editor: Nikos I. Karachalios

Copyright ( 2015 Arianna Dal Forno et al. This is an open access article distributed under the Creative Commons Attribution License, which permits unrestricted use, distribution, and reproduction in any medium, provided the original work is properly cited.

\begin{abstract}
In Braess paradox the addiction of an extra resource creates a social dilemma in which the individual rationality leads to collective irrationality. In the literature, the dynamics has been analyzed when considering impulsive commuters, i.e., those who switch choice regardless of the actual difference between costs. We analyze a dynamical version of the paradox with nonimpulsive commuters, who change road proportionally to the cost difference. When only two roads are available, we provide a rigorous proof of the existence of a unique fixed point showing that it is globally attracting even if locally unstable. When a new road is added the system becomes discontinuous and two-dimensional. We prove that still a unique fixed point exists, and its global attractivity is numerically evidenced, also when the fixed point is locally unstable. Our analysis adds a new insight in the understanding of dynamics in social dilemma.
\end{abstract}

\section{Introduction}

Assume that two different points of a network-an origin and a destination-are connected by two possible roads only. The Braess paradox states that, under specific conditions, adding a third road to the network decreases the efficiency of the network. This phenomenon is known in the transportation field and more general scientific literature (see [1-10]). Braess' paradox occurs because commuters try to minimize their own travel time ignoring the effect of their decisions on other commuters on the network. As a result, the total travel time may increase following an expansion of the network; in fact, even if some commuters are better off using the new link, they contribute to increase the congestion for other commuters.

The theoretical literature of the Braess paradox is particularly productive, especially in transportation, communication, and computer science (far from being exhaustive, see, e.g., $[10,11])$. Almost all the existing works have considered the basic network similar to the one presented in this paper, with the addition of a single link. Notably, [12] proposes a broader class of Braess graphs. A measure of the robustness of the dynamic network considering the influence of the flow on other links when certain component (node or link) is removed can be found in [13]. The empirical literature provides evidence in support of the paradox. For example, in [14] examples are reported that occurred on a modeled network of the city of Winnipeg, while [15] focus on a portion of the Boston road network. The experimental works are interested in studying the occurrence of the paradox in a controlled setting (see, e.g., [16-20]), not only in basic but also in augmented networks. This literature provides evidence in strong support of the paradox in some cases (see [18]), while statistically significant, but weaker support in some other cases (see $[16,17,19,20])$. A comparison of public versus private monitoring using the same participants is performed in [21] to investigate how the type of monitoring affects route choice. Interest in possible behaviors and composition of the population facing the basic network can be found in [22] which analyze the data gathered from the observation of an experiment with human participants, codes artificial 
behaviors emerged by mean of grounded theory, and uses ABM simulations. For a review on ABM with special focus on spacial interactions and networks see [23].

The paradox has also been presented in a dynamic game framework. A discrete time dynamic population model with social externalities and two available choices is studied in [24] simulating an adaptive adjustment process, and in particular with impulsive commuters in [25].

Following the clinical psychology literature (see [26]), impulsive commuters have been introduced in the analysis of binary choices with externalities in $[25,27]$ when considering commuters whose switching rate only depends on the sign of the difference between payoffs, no matter how much they differ. This approach has been used to consider choices both in small and in large groups in [28] and also when considering the introduction of a third choice which dramatically changes the dynamics in terms of complexity (as analyzed in [29]).

Analyzing the Braess paradox in terms of a ternary choice game shows new interesting dynamical characteristics that are investigated in [30], with a particular interest in the coexistence of several equilibria. Nevertheless, as illustrated in $[22,31]$, considering only impulsive commuters is not sufficient to describe the dynamics observed in experiments with human participants. Furthermore, as impulsive commuters consider only the sign and not the size of difference between costs, the population dynamics does not depend on the cost functions as long as the indifference point remains the same. In this paper, we consider a different behavior suggested by Amnon Rapoport (We are grateful to him for this helpful suggestion.) and which was used in [22] for artificial commuters. Although the full analysis of a homogenous-yet differentpopulation seems to provide a limited contribution, it may be a step forward to analyze the aggregate decision behavior in social dilemmas. In fact, in order to analyze heterogeneous populations, it is important to well understand the dynamics properties of component behaviors. In particular, we consider commuters which are concerned not only about the sign difference in payoffs but also on the relative difference. This kind of behavior is similar to the one considered in [32], where the propensity to switch choice is modulated by the difference between payoffs. Unlike the impulsive adjustment process, which makes the commuters change their choice as soon as a better choice occurs regardless of the difference, we consider a decision strategy which prescribes a change to the best available choice proportionally to the reported difference. Intuitively, this strategy seems to be more robust. The main result of the present work is that it is in fact robust. By contrast to the strategy considered in [30], which may lead to a stable cycle of any period, the strategy considered in this paper leads to a globally attracting fixed point. Depending on the parameters, this unique fixed point may be locally stable or unstable. However, the trajectories are convergent, in a few steps, to the fixed point. This result is rigorously proved for the case of a binary choice problem. For the ternary choice problem an analogous result is evidenced as well.

The plan of the work is as follows. In Section 2 the formalization of the dynamic model is reported. Section 2.1 includes the detailed description of the case with only two roads, which is represented by a one-dimensional piecewise smooth continuous map. We prove the existence of a unique fixed point, which may be locally stable or unstable in one partition. We prove that in any case it is globally attracting, that is, all the trajectories are ultimately converging to the fixed point. The case extended to three roads is considered in Section 3 and it is reduced to a two-dimensional piecewise smooth discontinuous map. We prove the existence of a unique fixed point, which may be locally attracting or not. We have only numerical evidence that also in this more general case, the fixed point is globally attracting. This can be rigorously proved for the particular cases in which the fixed point belongs to the boundaries of the domain of interest. The last section is devoted to the conclusions.

\section{The Dynamic Model with Two Roads}

The Braess paradox can be illustrated by Figure 1 as follows. Assume there is a unitary mass of commuters from start $(S)$ to end $(E)$ and there are two roads: one passing through left $(L)$ and the other through right $(R)$. The cost of each road is given by the sum of the time spent along each segment. In this network, segments $S-R$ and $L-E$ do not depend on traffic and they cost $a_{L}$ and $a_{R}$, respectively. On the contrary, time spent along segments $S-L$ and $R-E$ is proportional to the number of travellers and therefore, they cost $b_{L}(1-x)$ and $b_{R} x$, where $x \in[0,1]$ is the fraction of the population using segment $R-E$. At the Nash equilibrium commuters are distributed in such a way that both roads ( $S-L-E$ and $S-R-E$ ) have equal cos; that is, a fraction $\left(a_{R}+b_{R}-a_{L}\right) /\left(b_{L}+b_{R}\right)$ of commuters will choose $S-L-E$ and a fraction $\left(a_{L}+b_{L}-a_{R}\right) /\left(b_{L}+b_{R}\right)$ will choose $S-R$ $E$. For example, assume as in [22] that $a:=a_{L}=a_{R}=27$ and $b:=b_{L}=b_{R}=24$. Then, the symmetry of costs makes the population to split exactly into two equal fractions at the Nash equilibrium $\left(\left(a_{R}+b_{R}-a_{L}\right) /\left(b_{L}+b_{R}\right)=1 / 2\right)$, so that the travel time for both roads is $24(1 / 2)+27=39$. Now we assume that a very fast road is built connecting $L$ to $R$ at cost $d=3$. As we will see in Section 3, at the new Nash equilibrium commuters are distributed in such a way that the three roads $(S-L-E, S-L-R-E$, and $S-R-E)$ have equal cost; that is, a fraction $\left(d+b_{R}-a_{L}\right) / b_{R}$ of commuters will choose $S-L-E$, a fraction $\left(d+b_{L}-a_{R}\right) / b_{L}$ will choose $S-R-E$, and the rest will choose $S-L-R-E$. In the numerical example, this means that the entire population will choose $S-L-R-E$ (since in the other two roads the fraction of commuter is $(d+b-a) / b=0)$ with a total travel cost of $b_{L}+b_{R}+d=51$. According to [19], this is considered paradoxical as it shows how adding extra capacity to a network can reduce overall performance. More correctly, it is just counterintuitive and the mathematical reason is that there is a distinction between Nash equilibria and optima.

We consider the original network first made of two roads only (i.e., without the link $L-R$ ) as in Figure 1(a).

As in [24] this process can be modeled as a discrete time dynamical system considering commuters facing a binary choice between paths $S-L-E$ and $S-R-E$. For the sake of brevity these paths will be denoted as $L$ and $R$, respectively. To this 


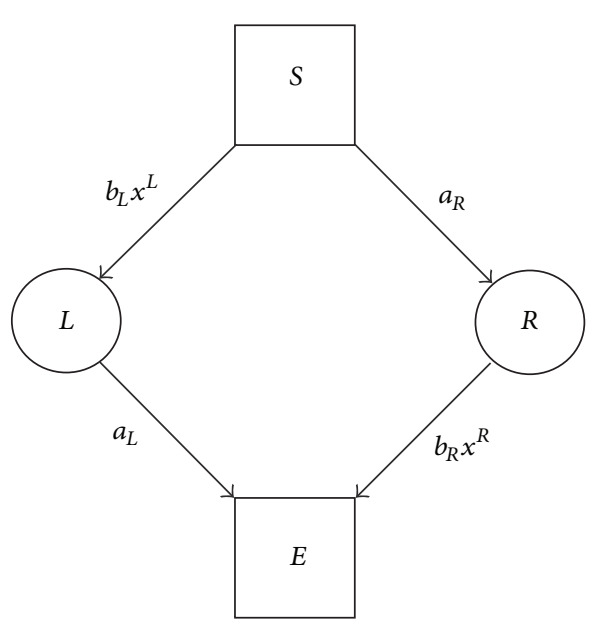

(a)

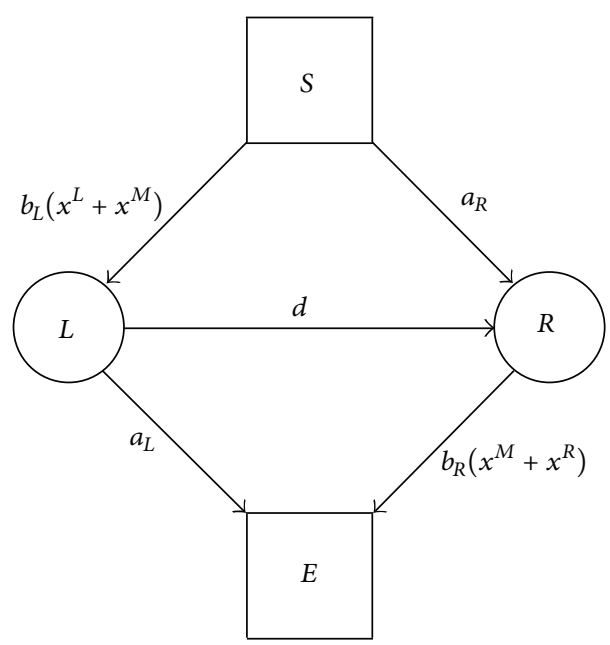

(b)

FIGURE 1: Basic network with the addition of the link from $L$ to $R$ in (b) and without in (a).

purpose, assume the set of commuters is normalized to the interval $[0,1]$. At each time period $t \geq 0, x_{t}^{R} \geq 0$ indicates the fraction of commuters choosing $R$ and $x_{t}^{L} \geq 0$ the fraction of those choosing $L$, given by $x_{t}^{L}=1-x_{t}^{R}$. Therefore, the travel times are given by

$$
\begin{aligned}
& L=a_{L}+b_{L} x^{L}, \\
& R=a_{R}+b_{R} x^{R},
\end{aligned}
$$

where $a_{L}, a_{R}, b_{L}, b_{R}>0$ and can be written as

$$
\begin{aligned}
& L\left(x^{R}\right)=a_{L}+b_{L}\left(1-x^{R}\right), \\
& R\left(x^{R}\right)=a_{R}+b_{R} x^{R} .
\end{aligned}
$$

This way, the travel times on the two roads are equal (and the commuters are indifferent) when $L\left(x^{R}\right)=R\left(x^{R}\right)$; that is, when $x^{R}=x^{*}$, where

$$
x^{*}=\frac{a_{L}+b_{L}-a_{R}}{b_{L}+b_{R}} .
$$

We say that the threshold $x^{*}$ is feasible when $x^{*} \in[0,1]$. However, $x^{*}=0$ (resp., $x^{*}=1$ ) clearly corresponds to the case in which the whole population chooses action $L$ (resp., $R$ ), and we shall see that this is indeed satisfied in the model described in the next subsection. We obtain the following constraints on the parameters in order to have $x^{*} \in(0,1)$, which we assume henceforth

$$
\begin{aligned}
& k_{1}=a_{L}+b_{L}-a_{R}>0, \\
& k_{2}=a_{R}+b_{R}-a_{L}>0 .
\end{aligned}
$$

2.1. The One-Dimensional PWS Map and Its Dynamics. Commuters are homogeneous and at each period decide their strategy considering the previous period travel costs assuming they will remain the same. At time $t+1$ the fraction
$x_{t}^{R}$ is common knowledge and each commuter observes the respective travel costs either $L\left(x_{t}^{R}\right)$ or $R\left(x_{t}^{R}\right)$, depending on their choice. The commuters decide their future action at time $t+1$ comparing the costs $L\left(x_{t}^{R}\right)$ and $R\left(x_{t}^{R}\right)$. We assume the commuters are nonimpulsive and myopically minimize their costs. That is, they change strategy proportionally to the difference between their chosen road and the best one, where the best road is the fastest. We introduce parameters $\delta_{L} \in(0,1)$ and $\delta_{R} \in(0,1)$ as switching rates, that is, the propensity for moving either to $L$ or to $R$, respectively. With nonimpulsive commuters these rates are adjusted by the normalized difference of travel costs. The difference $L\left(x_{t}^{R}\right)-$ $R\left(x_{t}^{R}\right)$ is normalized by the largest value of this difference, denoted by $k_{1}$ and obtained in correspondence of $x_{t}^{R}=0$. Therefore we have $k_{1}=L(0)-R(0)=a_{L}+b_{L}-a_{R}$ as already introduced in (4). Thus commuters are switching road proportionally to the side of the payoff difference. Similarly we can give a reason with respect to the difference $R\left(x_{t}^{R}\right)-L\left(x_{t}^{R}\right)$ to get a normalizing value. Now the largest value is obtained in correspondence of $x_{t}^{L}=0$; that is, $x_{t}^{R}=1$, and we get $k_{2}=R(1)-L(1)=a_{R}+b_{R}-a_{L}$ as already introduced in (4).

The resulting dynamics is $x_{t+1}^{R}=F\left(x_{t}^{R}\right)$ with $x_{t}^{R} \in[0,1]$ and the map $F:[0,1] \rightarrow[0,1]$ is defined as

$$
\begin{aligned}
& F\left(x^{R}\right) \\
& :=\left\{\begin{array}{l}
f_{L}\left(x^{R}\right) \\
=x^{R}+\delta_{R} \frac{L\left(x^{R}\right)-R\left(x^{R}\right)}{k_{1}}\left(1-x^{R}\right) \\
\text { if } 0 \leq x^{R} \leq x^{*} \\
f_{R}\left(x^{R}\right) \quad \text { if } x^{*} \leq x^{R} \leq 1 . \\
=x^{R}-\delta_{L} \frac{R\left(x^{R}\right)-L\left(x^{R}\right)}{k_{2}} x^{R}
\end{array}\right.
\end{aligned}
$$


Substituting from (2) and rearranging we obtain

$$
F\left(x^{R}\right):=\left\{\begin{aligned}
f_{L}\left(x^{R}\right)= & \delta_{R}+\left(1-\delta_{R}-\delta_{R} \frac{b_{L}+b_{R}}{k_{1}}\right) x^{R} \\
& +\delta_{R} \frac{b_{L}+b_{R}}{k_{1}}\left(x^{R}\right)^{2} \quad \text { if } 0 \leq x^{R} \leq x^{*} \\
f_{R}\left(x^{R}\right)= & \left(1+\delta_{L} \frac{k_{1}}{k_{2}}\right) x^{R} \\
& -\delta_{L} \frac{b_{L}+b_{R}}{k_{2}}\left(x^{R}\right)^{2} \quad \text { if } x^{*} \leq x^{R} \leq 1 .
\end{aligned}\right.
$$

From the definition of $x^{*}$ given in (3), it can also be written as

$$
x^{*}=\frac{a_{L}+b_{L}-a_{R}}{b_{L}+b_{R}}=\frac{k_{1}}{b_{L}+b_{R}}=\frac{k_{1}}{k_{1}+k_{2}},
$$

at which we have $L\left(x^{*}\right)=R\left(x^{*}\right)$, it is also $f_{L}\left(x^{*}\right)=f_{R}\left(x^{*}\right)$ and thus $F$ is continuous in $x^{*}$ and it is a fixed point as $F\left(x^{*}\right)=x^{*}$. We have the following proposition.

Proposition 1. Map $F$ is continuous in $[0,1]$ and under the conditions given in (4) $x^{*}=k_{1} /\left(b_{L}+b_{R}\right) \in(0,1)$ is the unique fixed point.

Proof. Only the uniqueness is left to prove. The equation $f_{L}\left(x^{R}\right)=x^{R}$ has two solutions: $x^{R}=x^{*}$ and $x^{R}=1$ which is larger than $x^{*}$ and thus a virtual fixed point. The equation $f_{R}\left(x^{R}\right)=x^{R}$ has two solutions: $x^{R}=x^{*}$ and $x^{R}=0$ which is smaller than $x^{*}$ and thus a virtual fixed point. Thus, the unique solution is $x^{R}=x^{*}$.

Clearly the fixed point may be locally stable or instable, depending on the slopes of the functions on the right and left sides of $x^{*}$. However, we shall see that in any case it is globally attracting. That is, any i.c. in the interval $[0,1]$ has a trajectory which converges to $x^{*}$.

Proposition 2. The fixed point $x^{*}=k_{1} /\left(b_{L}+b_{R}\right) \in(0,1)$ is globally attracting.

Proof. Even if $F\left(x^{R}\right)$ is not smooth in its fixed point (as indeed $x^{*}$ is a kink point of this map), the two components $f_{L}\left(x^{R}\right)$ and $f_{R}\left(x^{R}\right)$ are smooth in $x^{*}$ so that the left and right side derivatives of $F\left(x^{R}\right)$ in $x^{*}$ are well defined: $F_{-}^{\prime}\left(x^{*}\right)=$ $\lim _{x^{R} \rightarrow x^{*-}} F^{\prime}\left(x^{R}\right)=f_{L}^{\prime}\left(x^{*}\right)$ and $F_{+}^{\prime}\left(x^{*}\right)=\lim _{x^{R} \rightarrow x^{*+}} F^{\prime}\left(x^{R}\right)=$ $f_{R}^{\prime}\left(x^{*}\right)$.

$$
\begin{aligned}
& f_{L}^{\prime}\left(x^{R}\right)=\left(1-\delta_{R}-\delta_{R} \frac{b_{L}+b_{R}}{k_{1}}\right)+2 \delta_{R} \frac{b_{L}+b_{R}}{k_{1}} x^{R}, \\
& f_{L}^{\prime}\left(x^{*}\right)=1-\delta_{R} \frac{k_{2}}{k_{1}}
\end{aligned}
$$

while

$$
\begin{aligned}
& f_{R}^{\prime}\left(x^{R}\right)=\left(1+\delta_{L} \frac{k_{1}}{k_{2}}\right)-2 \delta_{L} \frac{b_{L}+b_{R}}{k_{2}} x^{R}, \\
& f_{R}^{\prime}\left(x^{*}\right)=1-\delta_{L} \frac{k_{1}}{k_{2}} .
\end{aligned}
$$

From

$$
f_{L}^{\prime \prime}\left(x^{R}\right)=2 \delta_{R} \frac{b_{L}+b_{R}}{k_{1}}>0, \quad f_{R}^{\prime \prime}\left(x^{R}\right)=-2 \delta_{L} \frac{b_{L}+b_{R}}{k_{2}}<0
$$

we have that on the left side of the fixed point the function is convex, while on the right side of the fixed point the function is concave.

Notice that $k_{2} / k_{1}$ only depends on $a_{L}, b_{L}, a_{R}, b_{R}$, and it is positive under our assumptions. Depending on the values of the ratio $k_{2} / k_{1}$ we can classify the right/left stability of the fixed point $x^{*}$. From (9) we clearly have always $f_{L}^{\prime}\left(x^{*}\right)<1$; thus the stability/instability on the left side of the fixed point depends on the condition $f_{L}^{\prime}\left(x^{*}\right)>-1$, which holds if and only if

$$
\frac{k_{2}}{k_{1}}<\frac{2}{\delta_{R}}
$$

Similarly, from (11) we clearly have always $f_{R}^{\prime}\left(x^{*}\right)<1$, and the stability/instability on the right side of the fixed point depends on the condition $f_{R}^{\prime}\left(x^{*}\right)>-1$, which holds if and only if

$$
\frac{k_{2}}{k_{1}}>\frac{\delta_{L}}{2}
$$

And clearly we have the following sequence of inequalities (to be used below):

$$
0<\frac{\delta_{L}}{2}<\delta_{L}<1<\frac{1}{\delta_{R}}<\frac{2}{\delta_{R}} .
$$

To investigate the local stability of the fixed point we consider three intervals for $k_{2} / k_{1}$ :

(i) $0<k_{2} / k_{1} \leq \delta_{L} / 2$

(ii) $\delta_{L} / 2<k_{2} / k_{1}<2 / \delta_{R}$

(iii) $2 / \delta_{R} \leq k_{2} / k_{1}$.

In case (i) it is $f_{R}^{\prime}\left(x^{*}\right) \leq-1$, moreover, it is easy to see that for any $x^{R} \in\left[x^{*}, 1\right]$ it is $f_{R}^{\prime}\left(x^{R}\right)<f_{R}^{\prime}\left(x^{*}\right)$ and thus the function on the right side of the fixed point is strictly decreasing and expansive: each point on the right side of the fixed point is mapped to the left side of the fixed point in one iteration. While on the left side of the fixed point the convex function $f_{L}\left(x^{R}\right)$ has $f_{L}^{\prime}\left(x^{*}\right)=1-\delta_{R}\left(k_{2} / k_{1}\right)>0$ (as this holds for $k_{2} / k_{1}<1 / \delta_{R}$ which is satisfied in this interval). This implies that the point of local minimum on the left side, say $x_{l, m}^{R}$, is smaller than the fixed point, $x_{l, m}^{R}<x^{*}$. Then the fixed point is unique and the map is a contraction in the interval $W_{\text {loc }}^{s}=\left[x_{l, m}^{R}, x^{*}\right]$, and an absorbing interval which is mapped into $W_{\text {loc }}^{s}$ in one iteration is given by $J=\left[x_{l, m}^{R}, f_{R}^{-1}\left(x_{l, m}^{R}\right)\right]$ when $f_{R}(1)=1-\delta_{L}<x_{l, m}^{R}$, or $J=\left[x_{l, m}^{R}, 1\right]$ when $f_{R}(1)=$ $1-\delta_{L} \geq x_{l, m}^{R}$. Any initial condition belonging to $[0,1] \backslash J$ has a trajectory which is mapped into $J$ in a finite number of iterations. Thus $x^{*}$ is globally attracting. An example is shown in Figure 2(a).

Similarly we can reason when $k_{2} / k_{1}$ belongs to the third interval. In fact, in case (iii) it is $f_{L}^{\prime}\left(x^{*}\right) \leq-1$, moreover, it is 


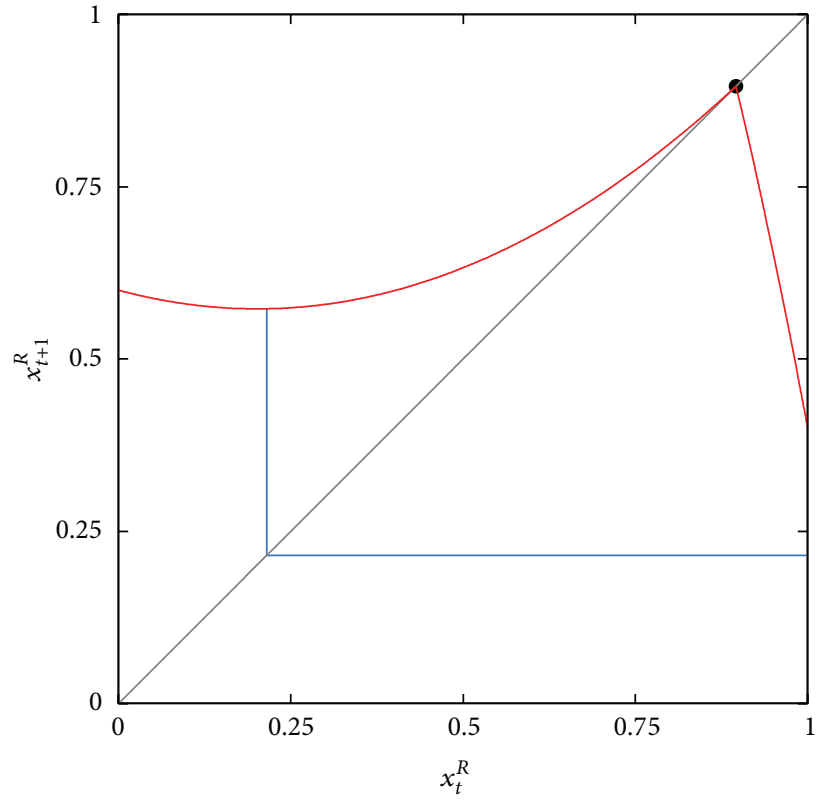

(a)

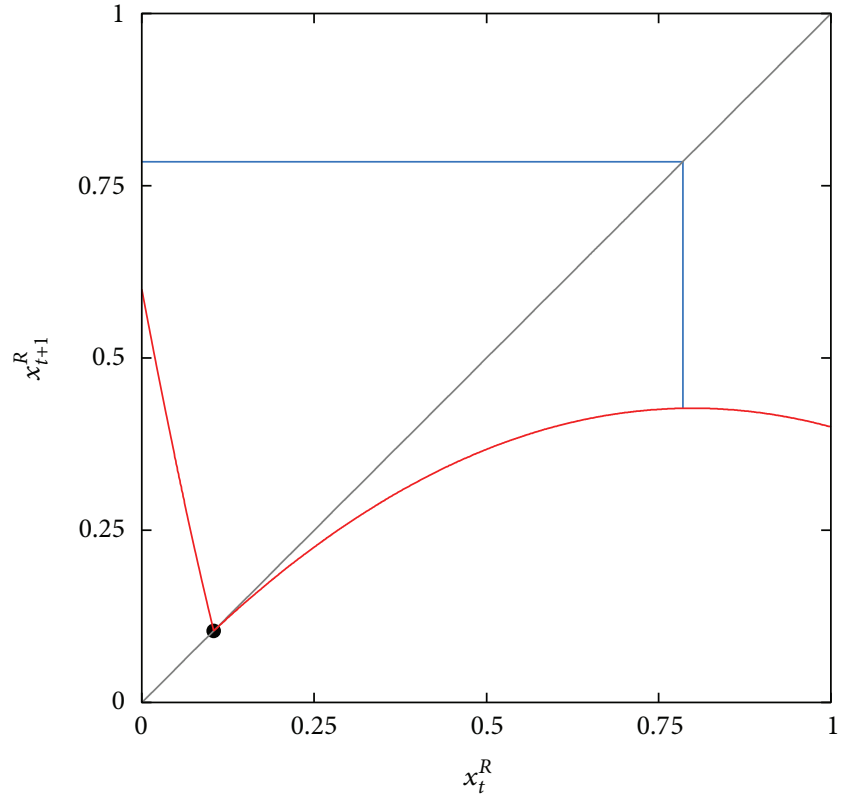

(b)

FIGURE 2: Qualitative shapes of the map. In (a) case (i), when $0<k_{2} / k_{1} \leq \delta_{L} / 2$, the fixed point is locally unstable on the right side. In (b) case (iii), when $2 / \delta_{R} \leq k_{2} / k_{1}$, the fixed point is locally unstable on the left side.

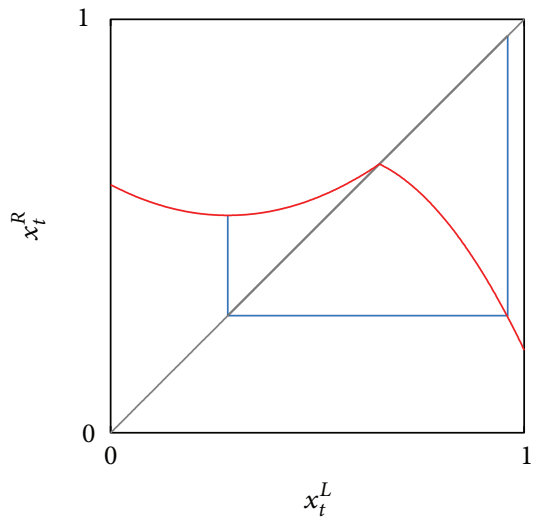

(a)

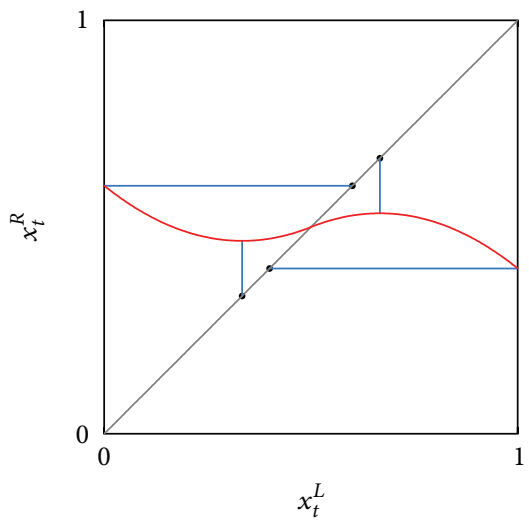

(b)

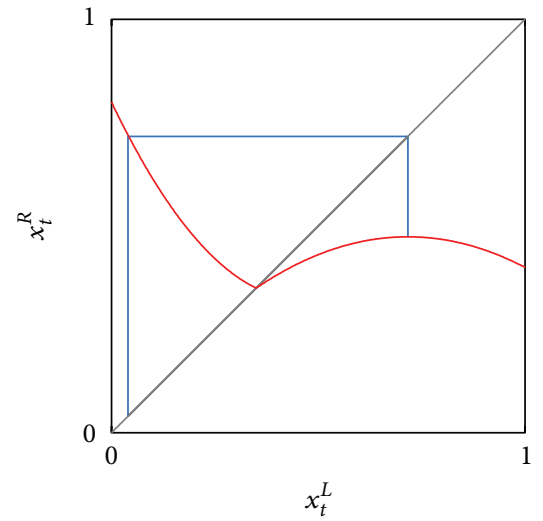

(c)

FIgure 3: Qualitative shapes of the map in case (ii), when $\delta_{L} / 2<k_{2} / k_{1} \leq 2 / \delta_{R}$ and the fixed point is locally stable on both sides.

easy to see that for any $x^{R} \in\left[0, x^{*}\right]$ it is $f_{L}^{\prime}\left(x^{*}\right)<f_{L}^{\prime}\left(x^{*}\right)$ and thus the function on the left side of the fixed point is strictly decreasing and expansive: each point on the left side of the fixed point is mapped to the right side of the fixed point in one iteration. While on the right side of the fixed point the concave function $f_{R}\left(x^{R}\right)$ has $f_{R}^{\prime}\left(x^{*}\right)=1-\delta_{L}\left(k_{1} / k_{2}\right)>0$ (as this holds for $k_{2} / k_{1}>\delta_{L}$ which is satisfied in this interval). This implies that the point of local maximum on the right side, say $x_{r, M}^{R}$, is larger than the fixed point, $x_{r, M}^{R}>x^{*}$. Then the map is a contraction in the interval $W_{\text {loc }}^{s}=\left[x^{*}, x_{r, M}^{R}\right]$, and an absorbing interval which is mapped into $W_{\text {loc }}^{s}$ in one iteration is given by $J=\left[f_{L}^{-1}\left(x_{r, M}^{R}\right), x_{r, M}^{R}\right]$ when $f_{L}(0)=$ $\delta_{R}>x_{r, M}^{R}$, or $J=\left[0, x_{r, M}^{R}\right]$ when $f_{L}(0)=\delta_{R} \leq x_{r, M}^{R}$. Any initial condition belonging to $[0,1] \backslash J$ has a trajectory which is mapped into $J$ in a finite number of iterations. Thus $x^{*}$ is globally attracting. An example is shown in Figure 2(b).

When $k_{2} / k_{1}$ belongs to the interval in (ii), $\delta_{L} / 2<k_{2} / k_{1}<$ $2 / \delta_{R}$, we have that the fixed point is locally stable on both sides. Moreover, inside this interval we can distinguish three regions, as already showed in (15), that is:

(a) $\delta_{L} / 2<k_{2} / k_{1} \leq \delta_{L}$ with $0<f_{L}^{\prime}\left(x^{*}\right)<1$ and $-1<f_{R}^{\prime}\left(x^{*}\right)<0$, as in Figure 3(a)

(b) $\delta_{L}<k_{2} / k_{1}<1 / \delta_{R}$ with $0<f_{L}^{\prime}\left(x^{*}\right)<1$ and $0<f_{R}^{\prime}\left(x^{*}\right)<1$, as in Figure 3(b) 
(c) $1 / \delta_{R} \leq k_{2} / k_{1}<2 / \delta_{R}$ with $-1<f_{L}^{\prime}\left(x^{*}\right)<0$ and $0<f_{R}^{\prime}\left(x^{*}\right)<1$, as in Figure 3(c).

In case (a) we have that the point of local minimum on the left side, say $x_{l, m}^{R}$, is smaller than the fixed point, $x_{l, m}^{R}<x^{*}$. Then the map is a contraction in the interval $W_{\mathrm{loc}}^{s}=\left[x_{l, m}^{R}, x^{*}\right]$, and on the right side the map is decreasing, so that an absorbing interval which is mapped into $W_{\text {loc }}^{s}$ in one iteration is given by $J=\left[x_{l, m}^{R}, f_{R}^{-1}\left(x_{l, m}^{R}\right)\right]$ when $f_{R}(1)=1-\delta_{L}<x_{l, m}^{R}$, or $J=\left[x_{l, m}^{R}, 1\right]$ when $f_{R}(1)=1-\delta_{L} \geq x_{l, m}^{R}$. Any initial condition belonging to $[0,1] \backslash J$ has a trajectory which is mapped into $J$ in a finite number of iterations. Thus $x^{*}$ is globally attracting.

In case (c) we can reason similarly, we have that the point of local maximum on the right side, say $x_{r, M}^{R}$, is larger than the fixed point, $x_{r, M}^{R}>x^{*}$. Then the map is a contraction in the interval $W_{\text {loc }}^{s}=\left[x^{*}, x_{r, M}^{R}\right]$, and on the left side the map is decreasing, so that an absorbing interval which is mapped into $W_{\text {loc }}^{s}$ in one iteration is given by $J=\left[f_{L}^{-1}\left(x_{r, M}^{R}\right), x_{r, M}^{R}\right]$ when $f_{L}(0)=\delta_{R}>x_{r, M}^{R}$, or $J=\left[0, x_{r, M}^{R}\right]$ when $f_{L}(0)=$ $\delta_{R} \leq x_{r, M}^{R}$. Any initial condition belonging to $[0,1] \backslash J$ has a trajectory which is mapped into $J$ in a finite number of iterations. Thus $x^{*}$ is globally attracting.

In case (b) we have that the point of local minimum on the left side, say $x_{l, m}^{R}$, is smaller than the fixed point, $x_{l, m}^{R}<$ $x^{*}$ and also that the point of local maximum on the right side, say $x_{r, M}^{R}$, is larger than the fixed point, $x_{r, M}^{R}>x^{*}$. Then the map is a contraction in the interval $W_{\text {loc }}^{s}=$ $\left[x_{l, m}^{R}, x_{r, M}^{R}\right]$ which includes the fixed point, and an absorbing interval which is mapped into $W_{\text {loc }}^{s}$ in one iteration is given by $J=\left[f_{L}^{-1}\left(x_{r, M}^{R}\right), f_{R}^{-1}\left(x_{l, m}^{R}\right)\right]$, or $J=\left[0, f_{R}^{-1}\left(x_{l, m}^{R}\right)\right]$ or $J=$ $\left[f_{L}^{-1}\left(x_{r, M}^{R}\right), 1\right]$ or $[0,1]$, depending under obvious conditions on the values of $\delta_{L}$ and $\delta_{R}$. When $J \subset[0,1]$ then any initial condition belonging to $[0,1] \backslash J$ has a trajectory which is mapped into $J$ in a finite number of iterations. Thus $x^{*}$ is globally attracting.

We notice that in Proposition 2 we prove that when the fixed point $x^{*}$ is internal to the interval $[0,1]$ then it is globally attracting. Let us turn to comment the two extrema, which occur when $x^{*}=0$ or $x^{*}=1$. These solutions clearly exist and are also globally attracting. In fact, the case $x^{*}=0$ corresponds to $k_{1}=L(0)-R(0)=a_{L}+b_{L}-a_{R}=0$ and only the function $f_{R}\left(x^{R}\right)$ is applied in the interval $[0,1]$ and from the concavity of the function we have that this fixed point is globally attracting even if the eigenvalue is equal to 1 . The other case $x^{*}=1$ corresponds to $k_{2}=R(1)-L(1)=a_{R}+b_{R}-a_{L}=0$, and only the function $f_{L}\left(x^{R}\right)$ is applied in the interval $[0,1]$ and from the convexity of the function we have that this fixed point is globally attracting even if the eigenvalue is equal to 1 .

\section{The Dynamics in the Expanded Network with Three Roads}

As it is well known, the Braess paradox introduces a new link and shows how this opportunity worsens both the individual and collective payoffs. This link introduces a new alternative: now the commuters are facing three choices, where the new one consists of path $S-L-R-E$ (see Figure 1(b)), which will be denoted as $M$ in the following. Models with three choices were introduced in [29] as it concerns discrete time and [33] as it concerns continuous time. In this paper we analyze the dynamics when the travel time $d$ for the new resulting link varies in a range that makes this link a dominant choice for some values and a dominated choice for others. It is worth to observe that in particular when the travel time on link $L-R$ is zero, the paradoxical result still holds.

Now assume that commuters switch road whenever travel time has become larger; that is, the other road is less costly and therefore more attractive. We indicate by $x^{L} \geq 0$ the fraction of population choosing action $L$, by $x^{M} \geq 0$ the fraction choosing action $M$, and by $x^{R} \geq 0$ the fraction choosing action $R$, with the constraint $x^{L}+x^{R}+x^{M}=1$. The travel times are given, respectively, as

$$
\begin{aligned}
& L=a_{L}+b_{L}\left(x^{L}+x^{M}\right), \\
& M=b_{L}\left(x^{L}+x^{M}\right)+b_{R}\left(x^{M}+x^{R}\right)+d, \\
& R=a_{R}+b_{R}\left(x^{M}+x^{R}\right) .
\end{aligned}
$$

By using the constraint let us define the fraction of population choosing $M$ as $x^{M}=1-x^{L}-x^{R} \geq 0$, so to consider only two variables, $\mathbf{x}=\left(x^{L}, x^{R}\right) \in \mathbb{R}_{+}^{2}$, and the phase space is the triangle

$$
D^{2}=\left\{\mathbf{x}=\left(x^{L}, x^{R}\right) \in \mathbb{R}_{+}^{2}: 0 \leq x^{L}+x^{R} \leq 1\right\}
$$

After substituting the expression of $x^{M}$ in the travel times we get

$$
\begin{aligned}
& L(\mathbf{x})=a_{L}+b_{L}\left(1-x^{R}\right) \\
& M(\mathbf{x})=b_{L}\left(1-x^{R}\right)+b_{R}\left(1-x^{L}\right)+d, \\
& R(\mathbf{x})=a_{R}+b_{R}\left(1-x^{L}\right) .
\end{aligned}
$$

The vertices of the phase space $P_{L}^{*}(1,0), P_{M}^{*}(0,0)$, and $P_{R}^{*}(0,1)$ represent states in which the whole population chooses, respectively, actions $L, M$, and $R$.

Since commuters are interested in minimizing travel time, we denote by $R_{A}$ the region in which the choice $A$ (where $A$ is $L, M$, or $R$ ) is preferable. These regions are called dominance regions of $A$. We have the following definition of the dominance regions:

$$
\begin{aligned}
R_{L} & =\left\{\mathbf{x} \in D^{2}: L(\mathbf{x}) \leq M(\mathbf{x}), L(\mathbf{x}) \leq R(\mathbf{x})\right\}, \\
R_{M} & =\left\{\mathbf{x} \in D^{2}: M(\mathbf{x}) \leq L(\mathbf{x}), M(\mathbf{x}) \leq R(\mathbf{x})\right\}, \\
R_{R} & =\left\{\mathbf{x} \in D^{2}: R(\mathbf{x}) \leq L(\mathbf{x}), \quad R(\mathbf{x}) \leq M(\mathbf{x})\right\} .
\end{aligned}
$$




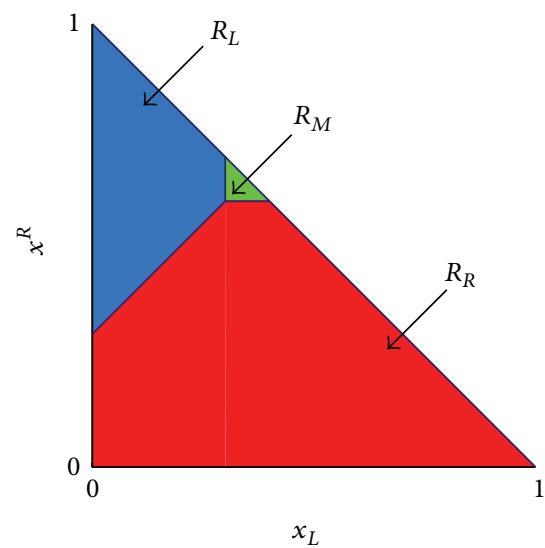

(a)

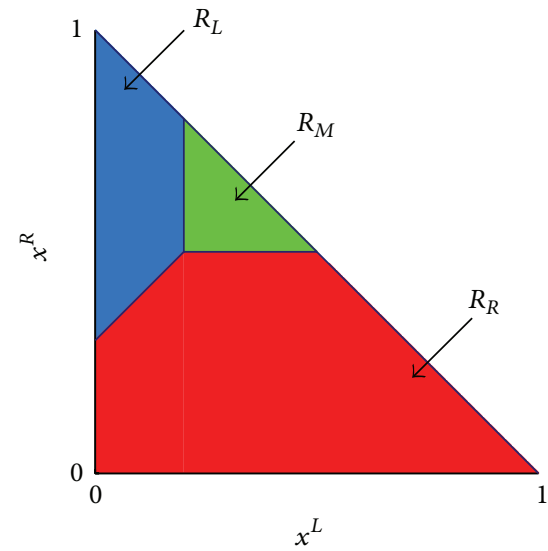

(b)

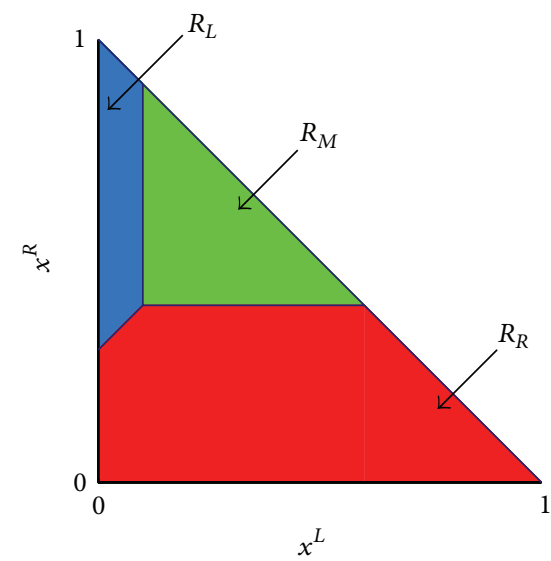

(c)

Figure 4: Dominance regions in the three choices network with different values of the cost parameter $d$ of the map $\overline{\mathbf{F}}$ : at $a_{L}=27, b_{L}=30$, $a_{R}=18, b_{R}=30$ and (a) $d=6$, (b) $d=3$, (c) $d=0$.

By using the expressions in (18) we get the following definitions for the regions

$$
\begin{aligned}
R_{L}=\{ & \mathbf{x} \in D^{2}: x^{L} \leq x^{* L}, \\
& \left.a_{L}+b_{L}\left(1-x^{R}\right) \leq a_{R}+b_{R}\left(1-x^{L}\right)\right\}, \\
R_{M}= & \left\{\mathbf{x} \in D^{2}: x^{L} \geq x^{* L}, x^{R} \geq x^{* R}\right\}, \\
R_{R}= & \left\{\mathbf{x} \in D^{2}: a_{R}+b_{R}\left(1-x^{L}\right) \leq a_{L}+b_{L}\left(1-x^{R}\right),\right. \\
& \left.x^{R} \leq x^{* R}\right\},
\end{aligned}
$$

where we have introduced

$$
\begin{aligned}
& x^{* L}=1-\frac{a_{L}-d}{b_{R}}=\frac{d-a_{L}+b_{R}}{b_{R}}, \\
& x^{* R}=1-\frac{a_{R}-d}{b_{L}}=\frac{d-a_{R}+b_{L}}{b_{L}} .
\end{aligned}
$$

The boundaries of the three regions are given by segments of straight lines of equations

$$
\begin{aligned}
& V_{1}: x^{L}=x^{* L}, \\
& V_{2}: x^{R}=x^{* R}, \\
& V_{3}: a_{L}+b_{L}\left(1-x^{R}\right)=a_{R}+b_{R}\left(1-x^{L}\right),
\end{aligned}
$$

and it is immediate to see that the point $\mathbf{x}^{*}=\left(x^{* L}, x^{* R}\right)$ satisfies also the third equation; that is, it belongs to all the three straight lines, $\mathbf{x}^{*} \in V_{i}$ for $i=1,2,3$.

Using the geometric representation in the triangle $D^{2}$ the dominance regions are illustrated in Figure 4 (via an example) using different colors. Three cases are shown, for different values of the parameter $d$, in order to illustrate how the region $R_{M}$ depends on the parameters of the third branch.

The point $\mathbf{x}^{*}=\left(x^{* L}, x^{* R}\right)$ is the unique point on the boundaries $V_{i}$ of all the three regions. Thus for our model we have to require $x^{* L} \geq 0, x^{* R} \geq 0$, and $x^{* L}+x^{* R} \leq 1$, leading to $x^{* M}=1-x^{* L}-x^{* R}$. In order to have three regions in $D^{2}$ it must be $x^{* L}>0, x^{* R}>0$, and $x^{* L}+x^{* R}<1$. The equality in one of these three conditions implies the disappearance of one region. For example, when $x^{* L}=0$, then $0 \leq x^{* R} \leq 1$ and $x^{* M}=1-x^{* R}$, leaving only the two regions $R_{R}$ and $R_{M}$ and so on.

The conditions $x^{* L} \geq 0, x^{* R} \geq 0$, and $x^{* L}+x^{* R} \leq 1$ lead to the constraints that will be assumed satisfied henceforth:

$$
\begin{gathered}
0 \leq \frac{a_{L}-d}{b_{R}} \leq 1, \quad 0 \leq \frac{a_{R}-d}{b_{L}} \leq 1, \\
\frac{a_{L}-d}{b_{R}}+\frac{a_{R}-d}{b_{L}} \geq 1 .
\end{gathered}
$$

We notice that from the third condition in (23) we have the values of $d$ for which the third region is really present in $D^{2}$; that is:

$$
0 \leq d \leq \frac{a_{L} b_{L}+a_{R} b_{R}-b_{L} b_{R}}{b_{R}+b_{L}} .
$$

Commuters are homogeneous and minimize their next period travel cost. At time $t+1$ the vector $\mathbf{x}_{t}$ becomes common knowledge, and each commuter can observe the travel costs $L\left(\mathbf{x}_{t}\right), M\left(\mathbf{x}_{t}\right)$, and $R\left(\mathbf{x}_{t}\right)$. We assume that if at time $t$ a fraction $x_{t}^{L}$ chooses path $L$, a fraction $x_{t}^{R}$ chooses action $R$ and travel costs are such that $R\left(\mathbf{x}_{t}\right)<L\left(\mathbf{x}_{t}\right)$ and $R\left(\mathbf{x}_{t}\right)<M\left(\mathbf{x}_{t}\right)$, then both a fraction of the $x_{t}^{L}$ commuters who chose action $L$ and a fraction of the $\left(1-x_{t}^{L}-x_{t}^{R}\right)$ commuters who chose action $M$ will switch to path $R$ in the next time period $t+1$. The fraction of switching commuters is given by the switching propensities $\delta_{L}, \delta_{M}, \delta_{R}$ modulated by the relative differences in payoffs. This happens whenever a path gives a smaller travel cost. In other words, at any time $t$ all the commuters decide their future action at time $t+1$ comparing the costs $L\left(\mathbf{x}_{t}\right), M\left(\mathbf{x}_{t}\right)$, and $R\left(\mathbf{x}_{t}\right)$. As in the one-dimensional case, here also the differences of travel costs are normalized dividing 
each difference by the constants giving the related maximum value, given in the different cases as follows:

$$
\begin{aligned}
k_{1} & =\max _{\mathbf{x}}[L(\mathbf{x})-R(\mathbf{x})] \\
& =[L(1,0)-R(1,0)]=a_{L}+b_{L}-a_{R}, \\
k_{2} & =\max _{\mathbf{x}}[R(\mathbf{x})-L(\mathbf{x})] \\
& =[R(0,1)-L(0,1)]=a_{R}+b_{R}-a_{L}, \\
k_{3} & =\max _{\mathbf{x}}[L(\mathbf{x})-M(\mathbf{x})] \\
& =\left[L\left(1, x^{R}\right)-M\left(1, x^{R}\right)\right]=a_{L}-d, \quad \forall x^{R} \in[0,1], \\
k_{4} & =\max _{\mathbf{x}}[M(\mathbf{x})-L(\mathbf{x})] \\
& =\left[M\left(0, x^{R}\right)-L\left(0, x^{R}\right)\right]=d+b_{R}-a_{L}, \quad \forall x^{R} \in[0,1], \\
k_{5} & =\max _{\mathbf{x}}[R(\mathbf{x})-M(\mathbf{x})] \\
& =\left[R\left(x^{L}, 1\right)-M\left(x^{L}, 1\right)\right]=a_{R}-d, \quad \forall x^{L} \in[0,1], \\
k_{6} & =\max _{\mathbf{x}}[M(\mathbf{x})-R(\mathbf{x})] \\
& =\left[M\left(x^{L}, 0\right)-R\left(x^{L}, 0\right)\right]=d+b_{L}-a_{R}, \quad \forall x^{L} \in[0,1] .
\end{aligned}
$$

The resulting dynamics are described by a two-dimensional map $\mathbf{x}_{t+1}=\overline{\mathbf{F}}\left(\mathbf{x}_{t}\right)$ defined as

$$
\overline{\mathbf{F}}: \mathbf{x}_{t+1}= \begin{cases}\mathbf{F}_{L}\left(\mathbf{x}_{t}\right) & \text { if } \mathbf{x}_{t} \in R_{L} \\ \mathbf{F}_{M}\left(\mathbf{x}_{t}\right) & \text { if } \mathbf{x}_{t} \in R_{M} \\ \mathbf{F}_{R}\left(\mathbf{x}_{t}\right) & \text { if } \mathbf{x}_{t} \in R_{R}\end{cases}
$$

where

$$
\begin{aligned}
& \mathbf{F}_{L}:\left\{\begin{array}{c}
x_{t+1}^{L}=x_{t}^{L}+\delta_{L}\left(\frac{R\left(\mathbf{x}_{t}\right)-L\left(\mathbf{x}_{t}\right)}{a_{R}+b_{R}-a_{L}} x_{t}^{R}\right. \\
\left.\quad+\frac{M\left(\mathbf{x}_{t}\right)-L\left(\mathbf{x}_{t}\right)}{d+b_{R}-a_{L}} x_{t}^{M}\right) \\
x_{t+1}^{R}=\left(1-\delta_{L} \frac{R\left(\mathbf{x}_{t}\right)-L\left(\mathbf{x}_{t}\right)}{a_{R}+b_{R}-a_{L}}\right) x_{t}^{R},
\end{array}\right. \\
& \mathbf{F}_{M}:\left\{\begin{array}{l}
x_{t+1}^{L}=\left(1-\delta_{M} \frac{L\left(\mathbf{x}_{t}\right)-M\left(\mathbf{x}_{t}\right)}{a_{L}-d}\right) x_{t}^{L} \\
x_{t+1}^{R}=\left(1-\delta_{M} \frac{R\left(\mathbf{x}_{t}\right)-M\left(\mathbf{x}_{t}\right)}{a_{R}-d}\right) x_{t}^{R},
\end{array}\right. \\
& \mathbf{F}_{R}:\left\{\begin{array}{c}
x_{t+1}^{L}=\left(1-\delta_{R} \frac{L\left(\mathbf{x}_{t}\right)-R\left(\mathbf{x}_{t}\right)}{a_{L}+b_{L}-a_{R}}\right) x_{t}^{L} \\
x_{t+1}^{R}=x_{t}^{R}+\delta_{R}\left(\frac{L\left(\mathbf{x}_{t}\right)-R\left(\mathbf{x}_{t}\right)}{a_{L}+b_{L}-a_{R}} x_{t}^{L}\right. \\
\left.+\frac{M\left(\mathbf{x}_{t}\right)-R\left(\mathbf{x}_{t}\right)}{d+b_{L}-a_{R}} x_{t}^{M}\right) .
\end{array}\right.
\end{aligned}
$$

By construction we have that $\overline{\mathbf{F}}$ maps $D^{2}$ into itself. In fact, to show that $\overline{\mathbf{F}}: D^{2} \rightarrow D^{2}$ it is enough to show that given $\left(x_{t}^{L}, x_{t}^{R}\right) \in D^{2}$ then $\left(x_{t+1}^{L}, x_{t+1}^{R}\right)=\overline{\mathbf{F}}\left(x_{t}^{L}, x_{t}^{R}\right) \in D^{2}$. From $\left(x_{t}^{L}, x_{t}^{R}\right) \in D^{2}$ we have that $0 \leq x_{t}^{L}+x_{t}^{R} \leq 1$ and $x_{t}^{L}+x_{t}^{R}+x_{t}^{M}=1$. Then if $\left(x_{t+1}^{L}, x_{t+1}^{R}\right)=\mathbf{F}_{L}\left(x_{t}^{L}, x_{t}^{R}\right)$, we have

$$
\begin{aligned}
x_{t+1}^{L}+x_{t+1}^{R} & =x_{t}^{L}+x_{t}^{R}+\delta_{L} \frac{M\left(\mathbf{x}_{t}\right)-L\left(\mathbf{x}_{t}\right)}{d+b_{R}-a_{L}} x_{t}^{M} \\
& \leq x_{t}^{L}+x_{t}^{R}+x_{t}^{M}=1,
\end{aligned}
$$

$x_{t+1}^{L} \geq 0$, and $x_{t+1}^{R} \geq 0$; thus $\left(x_{t+1}^{L}, x_{t+1}^{R}\right) \in D^{2}$. If $\left(x_{t+1}^{L}, x_{t+1}^{R}\right)=$ $\mathbf{F}_{M}\left(x_{t}^{L}, x_{t}^{R}\right)$ we have $0 \leq x_{t+1}^{L} \leq x_{t}^{L}$ and $0 \leq x_{t+1}^{R} \leq x_{t}^{R}$; thus $\left(x_{t+1}^{L}, x_{t+1}^{R}\right) \in D^{2}$. If $\left(x_{t+1}^{L}, x_{t+1}^{R}\right)=\mathbf{F}_{R}\left(x_{t}^{L}, x_{t}^{R}\right)$ we have

$$
\begin{aligned}
x_{t+1}^{L}+x_{t+1}^{R} & =x_{t}^{L}+x_{t}^{R}+\delta_{R} \frac{M\left(\mathbf{x}_{t}\right)-R\left(\mathbf{x}_{t}\right)}{d+b_{L}-a_{R}} x_{t}^{M} \\
& \leq x_{t}^{L}+x_{t}^{R}+x_{t}^{M}=1,
\end{aligned}
$$

$x_{t+1}^{L} \geq 0$, and $x_{t+1}^{R} \geq 0$; thus $\left(x_{t+1}^{L}, x_{t+1}^{R}\right) \in D^{2}$.

Substituting from (18) we can rewrite the functions in explicit form as

$$
\begin{aligned}
& \int x_{t+1}^{L}=\delta_{L}+\left(1-\delta_{L}-\delta_{L} \frac{b_{R}}{k_{4}}\right) x_{t}^{L} \\
& -\delta_{L} \frac{b_{L}}{k_{2}} x_{t}^{R}+\delta_{L} \frac{b_{L}}{k_{2}}\left(x_{t}^{R}\right)^{2} \\
& \mathbf{F}_{L}:\left\{\begin{aligned}
& +\delta_{L} b_{R}\left(\frac{1}{k_{4}}-\frac{1}{k_{2}}\right) x_{t}^{L} x_{t}^{R}+\delta_{L} \frac{b_{R}}{k_{4}}\left(x_{t}^{L}\right)^{2} \\
x_{t+1}^{R}= & \left(1-\delta_{L}-\delta_{L} \frac{b_{L}}{k_{2}}\right) x_{t}^{R} \\
& +\delta_{L} \frac{b_{R}}{k_{2}} x_{t}^{L} x_{t}^{R}-\delta_{L} \frac{b_{L}}{k_{2}}\left(x_{t}^{R}\right)^{2},
\end{aligned}\right. \\
& \mathbf{F}_{M}:\left\{\begin{array}{l}
x_{t+1}^{L}=\left(1+\delta_{M} \frac{k_{4}}{k_{3}}\right) x_{t}^{L}-\delta_{M} \frac{b_{R}}{k_{3}}\left(x_{t}^{L}\right)^{2} \\
x_{t+1}^{R}=\left(1+\delta_{M} \frac{k_{6}}{k_{5}}\right) x_{t}^{R}-\delta_{M} \frac{b_{L}}{k_{5}}\left(x_{t}^{R}\right)^{2},
\end{array}\right. \\
& \int x_{t+1}^{L}=\left(1-\delta_{R}-\delta_{R} \frac{b_{R}}{k_{1}}\right) x_{t}^{L} \\
& +\delta_{R} \frac{b_{L}}{k_{1}} x_{t}^{L} x_{t}^{R}-\delta_{R} \frac{b_{R}}{k_{1}}\left(x_{t}^{L}\right)^{2} \\
& \mathbf{F}_{R}:\left\{\begin{aligned}
x_{t+1}^{R}= & \delta_{R}+\left(1-\delta_{R}-\delta_{R} \frac{b_{L}}{k_{6}}\right) x_{t}^{R} \\
& -\delta_{R} \frac{b_{R}}{k_{1}} x_{t}^{L}+\delta_{R} \frac{b_{L}}{k_{1}}\left(x_{t}^{R}\right)^{2}
\end{aligned}\right. \\
& +\delta_{R} b_{L}\left(\frac{1}{k_{6}}-\frac{1}{k_{1}}\right) x_{t}^{L} x_{t}^{R}+\delta_{R} \frac{b_{R}}{k_{6}}\left(x_{t}^{L}\right)^{2} .
\end{aligned}
$$

Differently from the one-dimensional map $F$ considered in the previous section, the two-dimensional map $\overline{\mathbf{F}}$ is not continuous in $D^{2}$. More precisely, the functions $\mathbf{F}_{L}, \mathbf{F}_{M}$, and $\mathbf{F}_{R}$ are continuous, and thus $\overline{\mathbf{F}}$ is continuous in the interior of each region in which the different definitions apply. However, in general, except for the point $\mathbf{x}^{*}$, the map $\overline{\mathbf{F}}$ is not continuous along the borders $V_{i}, i=1,2,3$, of the regions $R_{L}, R_{M}$, and $R_{R}$. In fact, it is easy to see that considering 


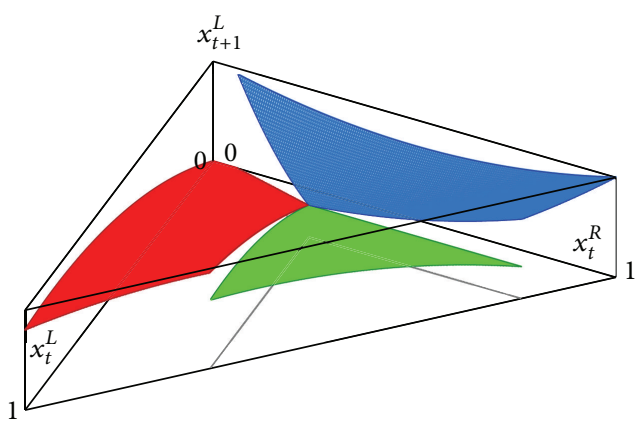

(a)

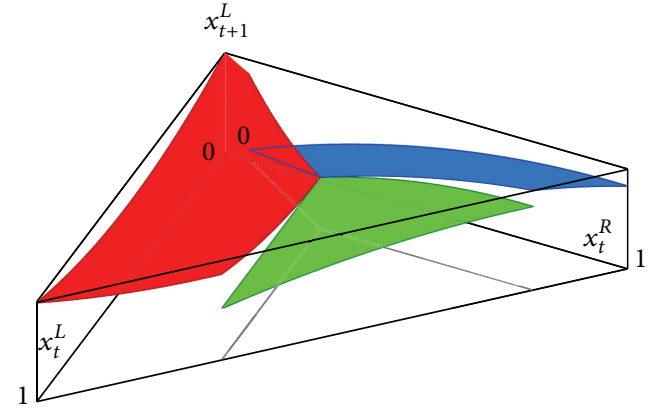

(b)

FIGURE 5: Graphics of the three functions defining (a) $x_{t+1}^{L}$ and (b) $x_{t+1}^{R}$, with parameters $a_{L}=29, b_{L}=16, a_{R}=19, b_{R}=24, d=8, \delta_{L}=0.5$, $\delta_{M}=0.8$, and $\delta_{R}=0.6$.

a point $\left(x^{L}, x^{R}\right)$ belonging to one of the lines defined in (22) at the boundary of two regions only, we have that the two functions defined on the two different regions lead to two different points of $D^{2}$. An example of how the three functions defining $x_{t+1}^{L}$ behave is shown in Figure 5(a), and the three functions defining $x_{t+1}^{R}$ are illustrated in Figure 5(b), showing that $\overline{\mathbf{F}}$ is continuous only in $\mathbf{x}^{*}$.

The point $\mathbf{x}^{*}$ in which the three regions are in contact and $\overline{\mathbf{F}}$ is continuous is clearly particular. In fact, solving the equation $L(\mathbf{x})=M(\mathbf{x})$ we have the unique solution $x^{L}=x^{* L}$ while solving the equation $R(\mathbf{x})=M(\mathbf{x})$ we have the unique solution $x^{R}=x^{* R}$. It follows that at the point $\mathbf{x}^{*}=\left(x^{* L}, x^{* R}\right)$ we have the same cost in each choice, as $L\left(\mathbf{x}^{*}\right)=M\left(\mathbf{x}^{*}\right)=$ $R\left(\mathbf{x}^{*}\right)$, so that at the point $\mathbf{x}^{*}$ the commuters are indifferent to any available choice and none of them will switch choice. Thus $\overline{\mathbf{F}}\left(\mathbf{x}^{*}\right)=\mathbf{x}^{*}$ and $\mathbf{x}^{*}$ is a stationary state. Under the conditions in (23) the point $\mathbf{x}^{*}$ is feasible, belonging to $D^{2}$. We can now prove that this is indeed the unique fixed point of map $\overline{\mathbf{F}}$.

Proposition 3. The point $\mathbf{x}^{*}=\left(x^{* L}, x^{* R}\right)$ given in (21) is the unique fixed point of the map $\overline{\mathbf{F}}$, belonging to $D^{2}$ when the conditions in (23) hold.

Proof. The proof of this proposition follows directly from the observation that

$$
\overline{\mathbf{F}}(\mathbf{x})=\mathbf{x} \text { if and only if } L(\mathbf{x})=M(\mathbf{x})=R(\mathbf{x}) .
$$

One way is already proved as we have detected the fixed point starting from the solution of $L(\mathbf{x})=M(\mathbf{x})$ and $R(\mathbf{x})=M(\mathbf{x})$, showing that it is the unique point $\mathbf{x}^{*}$. Vice versa, let us look for the solutions of $\overline{\mathbf{F}}(\mathbf{x})=\mathbf{x}$. We have the following systems (where $x^{M}=1-x^{L}-x^{R}$ ):

$$
\mathbf{F}_{L}:\left\{\begin{array}{c}
x^{L}=x^{L}+\delta_{L}\left(\frac{R(\mathbf{x})-L(\mathbf{x})}{k_{2}} x^{R}\right. \\
\left.+\frac{M(\mathbf{x})-L(\mathbf{x})}{k_{4}} x^{M}\right) \\
x^{R}=\left(1-\delta_{L} \frac{R(\mathbf{x})-L(\mathbf{x})}{k_{2}}\right) x^{R}
\end{array}\right.
$$$$
\text { if } \mathbf{x} \in R_{L} \text {, }
$$

$$
\begin{gathered}
\mathbf{F}_{M}:\left\{\begin{array}{c}
x^{L}=\left(1-\delta_{M} \frac{L(\mathbf{x})-M(\mathbf{x})}{a_{L}-d}\right) x^{L} \\
x^{R}=\left(1-\delta_{M} \frac{R(\mathbf{x})-M(\mathbf{x})}{a_{R}-d}\right) x^{R}
\end{array}\right. \\
\text { if } \mathbf{x} \in R_{M},
\end{gathered}
$$

or, equivalently (we recall that $\delta_{L}, \delta_{M}$, and $\delta_{R}$ are different from zero),

$\mathbf{F}_{L}:\left\{\begin{array}{c}\frac{R(\mathbf{x})-L(\mathbf{x})}{k_{2}} x^{R}+\frac{M(\mathbf{x})-L(\mathbf{x})}{k_{4}} x^{M}=0 \\ \frac{R(\mathbf{x})-L(\mathbf{x})}{k_{2}} x^{R}=0\end{array} \quad\right.$ if $\mathbf{x} \in R_{L}$,

$\mathbf{F}_{M}:\left\{\begin{array}{l}\frac{L(\mathbf{x})-M(\mathbf{x})}{a_{L}-d} x^{L}=0 \\ \frac{R(\mathbf{x})-M(\mathbf{x})}{a_{R}-d} x^{R}=0\end{array} \quad\right.$ if $\mathbf{x} \in R_{M}$,

$\mathbf{F}_{R}:\left\{\begin{array}{c}\frac{L(\mathbf{x})-R(\mathbf{x})}{k_{1}} x^{L}=0 \\ \frac{L(\mathbf{x})-R(\mathbf{x})}{k_{1}} x^{L}+\frac{M(\mathbf{x})-R(\mathbf{x})}{k_{6}} x^{M}=0\end{array} \quad\right.$ if $\mathbf{x} \in R_{R}$.

Considering the equation $\mathbf{F}_{L}(\mathbf{x})=\mathbf{x}$, we see that it is certainly satisfied for $x^{R}=0$ and $x^{M}=0$, which leads to the corner point with $x^{L}=1$. However, if the fixed point is an 
interior point of $D^{2}$ then the point $(1,0)$ does not belong to the proper region $R_{L}$; that is, it is a virtual fixed point. Clearly this equation is satisfied when $L(\mathbf{x})=M(\mathbf{x})$ and $L(\mathbf{x})=R(\mathbf{x})$, which occurs only at the point $\mathbf{x}^{*}$. It is possible to have $\mathbf{x}^{*}=$ $(1,0)$ and this occurs for $\left(a_{L}-d\right) / b_{R}=0$ and $\left(a_{R}-d\right) / b_{L}=1$. Thus we can state that the only solution of $\mathbf{F}_{L}(\mathbf{x})=\mathbf{x}$ in $R_{L}$ is given by the fixed point $\mathbf{x}^{*}$. Similarly we can reason in the other regions.

It is worth to mention that particular cases are obtained when the fixed point $\mathbf{x}^{*}$ belongs to a boundary of the region $D^{2}$. For example, when $x^{* R}+x^{* L}=1$ and $x^{* M}=0$, then only the two regions $R_{L}$ and $R_{R}$ are involved. This occurs when the parameters satisfy

$$
\begin{gathered}
d=\frac{a_{L} b_{L}+a_{R} b_{R}-b_{L} b_{R}}{b_{R}+b_{L}}, \quad 0 \leq \frac{a_{L}-d}{b_{R}} \leq 1, \\
0 \leq \frac{a_{R}-d}{b_{L}} \leq 1 .
\end{gathered}
$$

From $\left(a_{R}-d\right) / b_{L}=\left(a_{R}+b_{R}-a_{L}\right) /\left(b_{R}+b_{L}\right)$ we have $x^{* R}=$ $1-\left(a_{R}-d\right) / b_{L}=\left(a_{L}+b_{L}-a_{R}\right) /\left(b_{R}+b_{L}\right)=k_{1} /\left(b_{R}+b_{L}\right)=x^{*}$ as in the one-dimensional map studied in Section 2, and $x^{* L}=$ $1-x^{* R}=1-\left(a_{L}-d\right) / b_{R}=k_{2} /\left(b_{R}+b_{L}\right)=1-k_{1} /\left(b_{R}+b_{L}\right)=$ $1-x^{*}$. From the maps $\mathbf{F}_{L}$ and $\mathbf{F}_{R}$ in (27) and for the parameters occurring in this case, it can be seen that $x_{t+1}^{L}=1-x_{t+1}^{R}$ holds in both, so that the dynamics can be studied by using only the variable $x_{t}^{R}$ and the resulting one-dimensional map is as follows:

$$
\begin{aligned}
& F\left(x^{R}\right) \\
& := \begin{cases}f_{L}\left(x^{R}\right) & \text { if } 0 \leq x^{R} \leq x^{*} \\
=x^{R}+\delta_{R} \frac{L\left(x^{R}\right)-R\left(x^{R}\right)}{k_{1}}\left(1-x^{R}\right) & \\
f_{R}\left(x^{R}\right) \quad & \text { if } x^{*} \leq x^{R} \leq 1, \\
=x^{R}-\delta_{L} \frac{R\left(x^{R}\right)-L\left(x^{R}\right)}{k_{2}} x^{R} & \end{cases}
\end{aligned}
$$

which corresponds to the system already studied in Section 2. As we have seen, this fixed point is globally attracting, even if locally we can have the eigenvalue on some side larger than 1 in modulus.

The case with $x^{* L}=0$, so that $0 \leq x^{* R} \leq 1$ and $x^{* M}=$ $1-x^{* R}$, leaves only the two regions $R_{R}$ and $R_{M}$. This occurs when the parameters satisfy

$$
\frac{a_{L}-d}{b_{R}}=1, \quad 0 \leq \frac{a_{R}-d}{b_{L}} \leq 1 .
$$

From the maps $\mathbf{F}_{M}$ and $\mathbf{F}_{R}$ in (27) it can be seen that $x_{t}^{L}$ converges to $x^{* L}=0$ so that the resulting asymptotic dynamic behavior can be studied via the one-dimensional map given by $x_{t+1}^{R}=T\left(x_{t}^{R}\right)$ with $x_{t}^{R} \in[0,1]$ (and $x_{t}^{M}=1-x_{t}^{R}$ ), given by

$T\left(x^{R}\right)$

$$
:=\left\{\begin{array}{l}
T_{L}\left(x^{R}\right) \quad \text { if } 0 \leq x^{R} \leq x^{*} \\
=x^{R}+\delta_{R} \frac{M\left(x^{R}\right)-R\left(x^{R}\right)}{k_{6} \quad\left(1-x^{R}\right)} \\
T_{R}\left(x^{R}\right) \quad \text { if } x^{*} \leq x^{R} \leq 1, \\
=x^{R}-\delta_{M} \frac{R\left(x^{R}\right)-M\left(x^{R}\right)}{a_{R}-d} x^{R} \quad
\end{array}\right.
$$

where $M\left(x^{R}\right)-R\left(x^{R}\right)=a_{L}+b_{L}\left(1-x^{R}\right)-a_{R}-b_{R}$.

The case with $x^{* R}=0$, so that $0 \leq x^{* L} \leq 1$ and $x^{* M}=1-x^{* L}$, leaves only the two regions $R_{L}$ and $R_{M}$. This occurs when the parameters satisfy $0 \leq\left(a_{L}-d\right) / b_{R} \leq 1$, $\left(a_{R}-d\right) / b_{L}=1$. From the maps $\mathbf{F}_{M}$ and $\mathbf{F}_{L}$ in (27) it can be seen that $x_{t}^{R}$ converges to $x^{* R}=0$ so that the resulting asymptotic dynamic behavior can be studied via the onedimensional map given by $x_{t+1}^{L}=T\left(x_{t}^{L}\right)$ similar to the one obtained in (37) with obvious changes.

By using arguments as in Section 2, it can be shown that these one-dimensional systems have a unique fixed point which is globally attracting (although it can be locally stable or unstable).

In order to study the local stability of the fixed point of the two-dimensional map $\overline{\mathbf{F}}$, we can use the linearization of the different definitions in the fixed point, which leads to the following Jacobian matrices, one for each region $R_{L}, R_{M}$, and $R_{R}$, that we denote, respectively, with $J_{L}, J_{M}$, and $J_{R}$ :

$$
\begin{aligned}
& J_{L}\left(\mathbf{x}^{*}\right)=\left(\begin{array}{cc}
1+\delta_{L}-\delta_{L} \frac{b_{R}}{b_{L}} \frac{k_{5}}{k_{4}}-\delta_{L} \frac{b_{R}}{b_{L}} \frac{k_{6}}{k_{1}} & \delta_{L} \frac{k_{6}}{k_{1}} \\
\delta_{L} \frac{b_{R}}{b_{L}} \frac{k_{6}}{k_{1}} & 1-\delta_{L} \frac{k_{6}}{k_{1}}
\end{array}\right), \\
& J_{M}\left(\mathbf{x}^{*}\right)=\left(\begin{array}{cc}
1-\delta_{M} \frac{k_{4}}{k_{3}} & 0 \\
0 & 1-\delta_{M} \frac{k_{6}}{k_{5}}
\end{array}\right), \\
& J_{R}\left(\mathbf{x}^{*}\right)=\left(\begin{array}{cc}
1-\delta_{R} \frac{k_{5}}{k_{2}} & \delta_{R} \frac{b_{L}}{b_{R}} \frac{k_{5}}{k_{2}} \\
\delta_{R} \frac{k_{5}}{k_{2}} & 1+\delta_{R}-\delta_{R} \frac{b_{L}}{b_{R}} \frac{k_{3}}{k_{6}}-\delta_{R} \frac{b_{L}}{b_{R}} \frac{k_{5}}{k_{2}}
\end{array}\right) .
\end{aligned}
$$

The two real eigenvalues of the Jacobian matrix in $R_{M}$ are in explicit form and both are smaller than +1 . However, they can be larger than -1 or not. Also the eigenvalues of the other jacobian matrices can be in modulus smaller or larger than 1. However, the global dynamics observed numerically give always trajectories which are convergent to the fixed point $\mathbf{x}^{*}$, at any set of allowed parameters values. 


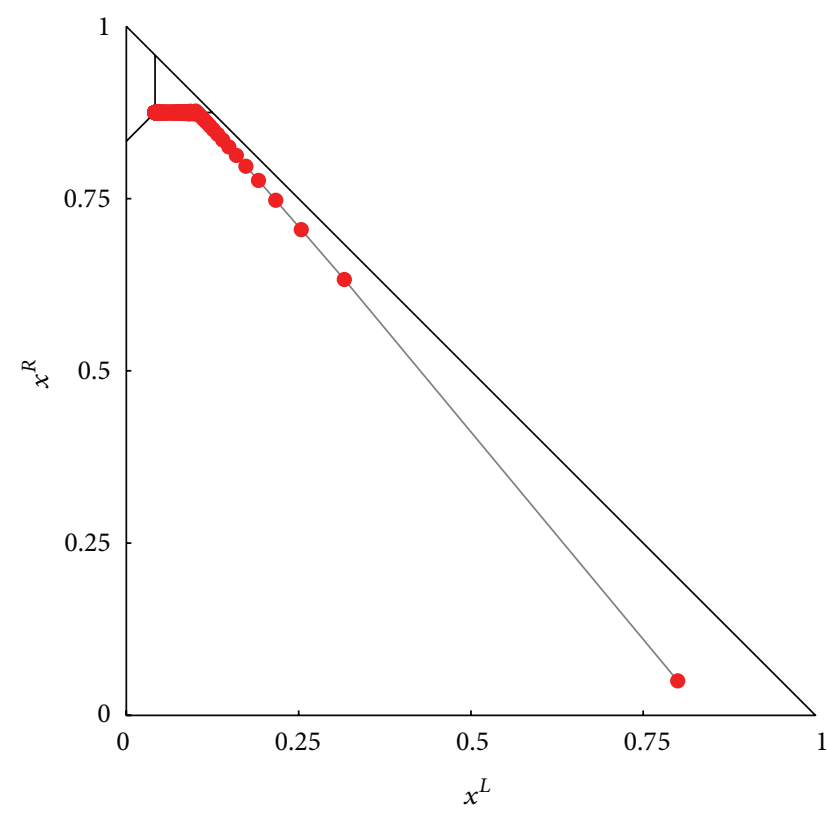

(a)

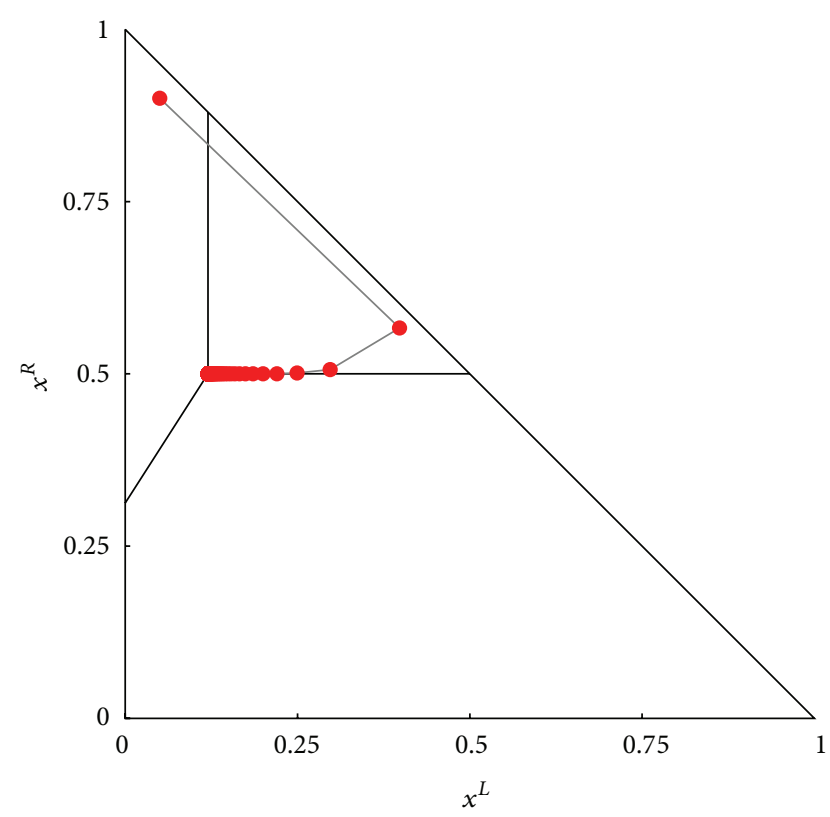

(b)

FIGURE 6: Examples of trajectories converging to the unique fixed point with parameters (a) $a_{L}=28, b_{L}=24, a_{R}=8, b_{R}=24, d=5, \delta_{L}=0.4$, $\delta_{M}=0.3, \delta_{R}=0.7$ and i.c. $x^{L}=0.8, x^{R}=0.05$; (b) $a_{L}=29, b_{L}=16, a_{R}=15, b_{R}=25, d=7, \delta_{L}=0.5, \delta_{M}=0.8, \delta_{R}=0.6$ and i.c. $x^{L}=0.05$, $x^{R}=0.9$. Dots are linked by a continuous line for illustrative purpose only.

This global attractivity in the two-dimensional map is difficult to be proved rigorously; however the results in the particular cases commented above, that is, when the fixed point $\mathbf{x}^{*}$ belongs to the boundary of $D^{2}$, suggests that the same result is true also when the fixed point is in the interior of $D^{2}$.

In Figure 6 we show some examples: in Figure 6(a) we can see a trajectory starting at $R_{R}$; in Figure 6(b) a trajectory whose initial point in $R_{L}$ is mapped into $R_{M}$. The shape of the functions in the case of Figure 6(b) is similar to the one shown in Figure 5.

\section{Conclusion}

In this paper we have analyzed a dynamical version of the Braess paradox with nonimpulsive commuters, who change road proportionally to the cost difference. We were interested to prove that with nonimpulsive commuters there exists a unique equilibrium both in the original network with two choices and in the ternary one, when a single link is added. We have shown that both models are quite robust. We were able to provide an analytical proof of the global attractivity of the unique fixed point in the one-dimensional case, when only two roads are available and we used numerical techniques and simulations to give evidence in the two-dimensional case (i.e., when a new road is added), as a rigorous proof can be done only for particular border cases.

A limitation of our contribution is considering homogeneous populations in spite of the fact that the evidence supports heterogeneity. Nevertheless, heterogeneity of commuters' behavior cannot be easily classified; yet, even when conducting experiments, common individual patterns are just identified and classified. On the other hand, when observing the aggregate behavior, in many cases the literature provides evidence in support of the paradox outcome, that is, the convergence to the Nash equilibrium. The impulsive agents in [30] react faster than the proportional agents presented in this paper; yet, as a population they fail to achieve the Nash equilibria in certain cases, while in this paper the aggregate behavior does not. We proposed this aggregate behavior because it replies the convergence to the Nash equilibria and at the same time it may be the result of modeling some observed individual behaviors, such as imitation and free riding.

Taking into account the limitation of homogeneous population analysis, a natural extension of our contribution is considering heterogeneous populations with both impulsive and nonimpulsive commuters. We have reasons to believe that in such a case some properties of the different behaviors are inherited and some are lost. Therefore the results we provide in this paper match those for impulsive populations and are paramount to extend the analysis to more realistic cases. In fact-as the empirical results show-heterogeneous populations dynamics provide a better fit of what has been observed when considering human participants interaction.

Furthermore, it will be interesting to consider generalizations to networks with richer architectures. Finally, we observe that, since with the class of behavior we consider in this paper that dynamics depends on the shape of the cost functions, it will be interesting to extend the analysis to nonlinear cost functions. 


\section{Conflict of Interests}

The authors declare that there is no conflict of interests regarding the publication of this paper.

\section{Acknowledgments}

V. Avrutin is supported by the European Community within the scope of the project "Multiple-discontinuity induced bifurcations in theory and applications" (Marie Curie Action of the 7th Framework Programme, Contract Agreement no. PIEF-GA-2011-300281). U. Merlone has worked under the auspices of COST Action IS1104 "The EU in the new complex geography of economic systems: models, tools, and policy evaluation."

\section{References}

[1] R. Arnott and K. Small, "The economics of traffic congestion," American Scientist, vol. 82, no. 5, pp. 446-455, 1994.

[2] T. Bass, "Road to ruin,” Discover, pp. 56-61, 1992.

[3] B. Calvert and G. Keady, "Braess's paradox and power-law nonlinearities in networks," The Journal of the Australian Mathematical Society B: Applied Mathematics, vol. 35, no. 1, pp. 1-22, 1993.

[4] J. E. Cohen and C. Jeffries, "Congestion resulting from increased capacity in single-server queueing networks," IEEE/ACM Transactions on Networking, vol. 5, no. 2, pp. 305-310, 1997.

[5] J. E. Cohen and F. P. Kelly, "A paradox of congestion in a queuing network," Journal of Applied Probability, vol. 27, no. 3, pp. 730734,1990 .

[6] S. Dafermos and A. Nagurney, "On some traffic equilibrium theory paradoxes," Transportation Research B: Methodological, vol. 18, no. 2, pp. 101-110, 1984.

[7] C. Fisk, "More paradoxes in the equilibrium assignment problem," Transportation Research B: Methodological, vol. 13, no. 4, pp. 305-309, 1979.

[8] W. H. K. Lam, "Effects of road pricing on system performance," Traffic Engineering \& Control, vol. 29, no. 12, pp. 631-635, 1988.

[9] J. D. Murchland, "Braess's paradox of traffic flow," Transportation Research, vol. 4, no. 4, pp. 391-394, 1970.

[10] R. Steinberg and W. I. Zangwill, “The prevalence of Braess' paradox," Transportation Science, vol. 17, no. 3, pp. 301-318, 1983.

[11] M. Frank, "The Braess paradox," Mathematical Programming, vol. 20, no. 1, pp. 283-302, 1981.

[12] T. Roughgarden, Selfish Routing and the Price of Anarchy, MIT press, 2005.

[13] B. B. Fu, C. X. Zhao, and S. B. Li, "The analysis of Braess' paradox and robustness based on dynamic traffic assignment models," Discrete Dynamics in Nature and Society, vol. 2013, Article ID 796842, 8 pages, 2013.

[14] C. Fisk and S. Pallottino, "Empirical evidence for equilibrium paradoxes with implications for optimal planning strategies," Transportation Research Part A: General, vol. 15, no. 3, pp. 245248, 1981.

[15] H. Youn, M. T. Gastner, and H. Jeong, "Price of anarchy in transportation networks: efficiency and optimality control," Physical Review Letters, vol. 101, no. 12, Article ID 128701, 2008.

[16] K. Aoki, Y. Ohtsubo, A. Rapoport, and T. Saijo, "Effects of prior investment and personal responsibility in a simple network game," Current Research in Social Psychology, vol. 13, no. 2, pp. 10-21, 2007.

[17] J. Morgan, H. Orzen, and M. Sefton, "Network architecture and traffic flows: experiments on the Pigou-Knight-Downs and Braess paradoxes," Games and Economic Behavior, vol. 66, no. 1, pp. 348-372, 2009.

[18] A. Rapoport, V. Mak, and R. Zwick, "Navigating congested networks with variable demand: experimental evidence," Journal of Economic Psychology, vol. 27, no. 5, pp. 648-666, 2006.

[19] A. Rapoport, T. Kugler, S. Dugar, and E. J. Gisches, "Braess paradox in the laboratory: an experimental study of route choice in traffic networks with asymmetric costs," in Decision Modeling and Behavior in Uncertain and Complex Environment, T. Kugler, J. C. Smith, T. Connolly, and Y. J. Son, Eds., pp. 309-337, Springer, New York, NY, USA, 2008.

[20] A. Rapoport, T. Kugler, S. Dugar, and E. J. Gisches, "Choice of routes in congested traffic networks: experimental tests of the Braess paradox," Games and Economic Behavior, vol. 65, no. 2, pp. 538-571, 2009.

[21] E. J. Gisches and A. Rapoport, "Degrading network capacity may improve performance: private versus public monitoring in the Braess paradox," Theory and Decision, vol. 73, no. 2, pp. 267293, 2012.

[22] A. Dal Forno and U. Merlone, "Replicating human interaction in Braess paradox," in Proceedings of the Simulation Conference (WSC '13), pp. 1754-1765, IEEE, 2013.

[23] M. Ausloos, H. Dawid, and U. Merlone, "Spatial interactions in agent-based modeling," in Complexity and Geographical Economics: Topics and Tools, P. Commendatore, S. S. Kayam, and I. Kubin, Eds., Springer, Berlin, Germany, 2014.

[24] G.-I. Bischi and U. Merlone, "Global dynamics in binary choice models with social influence," The Journal of Mathematical Sociology, vol. 33, no. 4, pp. 277-302, 2009.

[25] G. I. Bischi, L. Gardini, and U. Merlone, "Impulsivity in binary choices and the emergence of periodicity," Discrete Dynamics in Nature and Society, vol. 2009, Article ID 407913, 22 pages, 2009.

[26] J. H. Patton, M. S. Stanford, and E. S. Barratt, "Factor structure of the Barratt Impulsiveness Scale," Journal of Clinical Psychology, vol. 51, no. 6, pp. 768-774, 1995.

[27] G. I. Bischi, L. Gardini, and U. Merlone, "Periodic cycles and bifurcation curves for one-dimensional maps with two discontinuities," Journal of Dynamical Systems Geometric Theories, vol. 7, no. 2, pp. 101-123, 2009.

[28] G.-I. Bischi and U. Merlone, "Binary choices in small and large groups: a unified model," Physica A, vol. 389, no. 4, pp. 843-853, 2010.

[29] A. Dal Forno, L. Gardini, and U. Merlone, "Ternary choices in repeated games and border collision bifurcations," Chaos, Solitons \& Fractals, vol. 45, no. 3, pp. 294-305, 2012.

[30] A. Dal Forno and U. Merlone, "Border-collision bifurcations in a model of Braess paradox," Mathematics and Computers in Simulation, vol. 87, pp. 1-18, 2013.

[31] A. Dal Forno, V. Giorgino, and U. Merlone, "Agent-based modeling with GT methodology. An example," Working Paper, 2014.

[32] L. Gardini, U. Merlone, and F. Tramontana, "Inertia in binary choices: continuity breaking and big-bang bifurcation points," Journal of Economic Behavior \& Organization, vol. 80, no. 1, pp. 153-167, 2011.

[33] W. H. Sandholm, Population Games and Evolutionary Dynamics, The MIT Press, Cambridge, Mass, USA, 2010. 


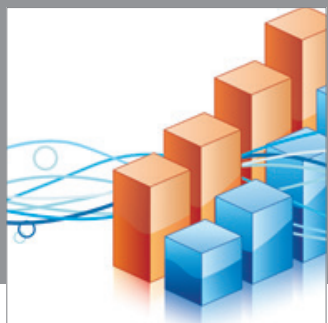

Advances in

Operations Research

mansans

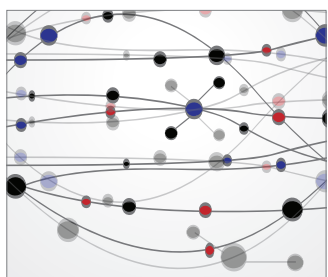

The Scientific World Journal
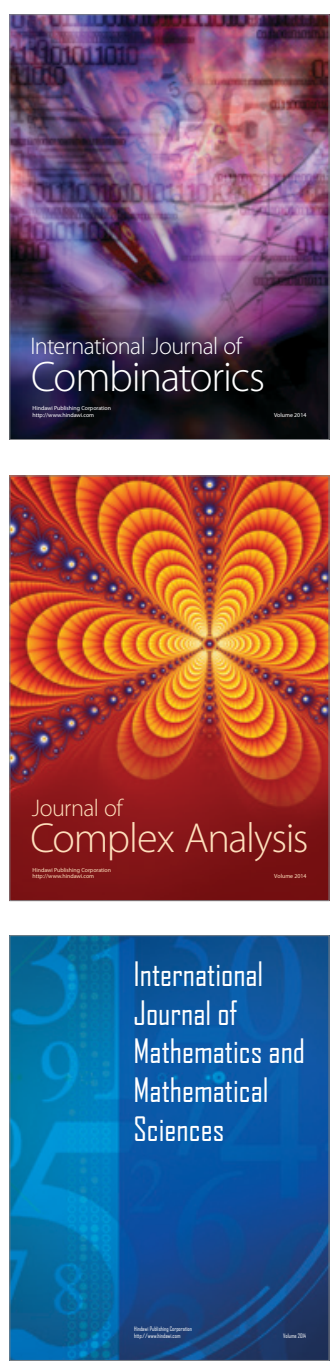
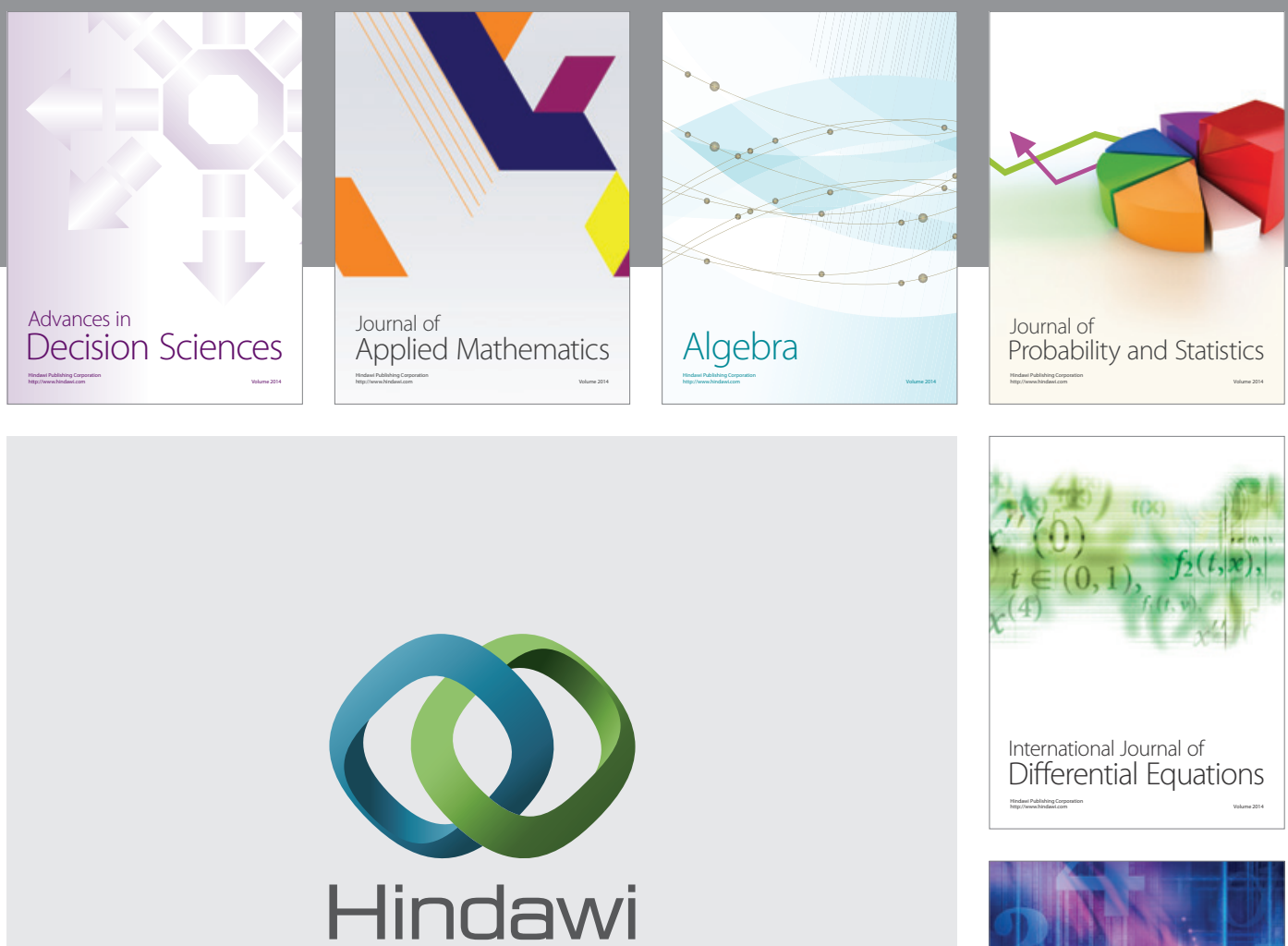

Submit your manuscripts at http://www.hindawi.com
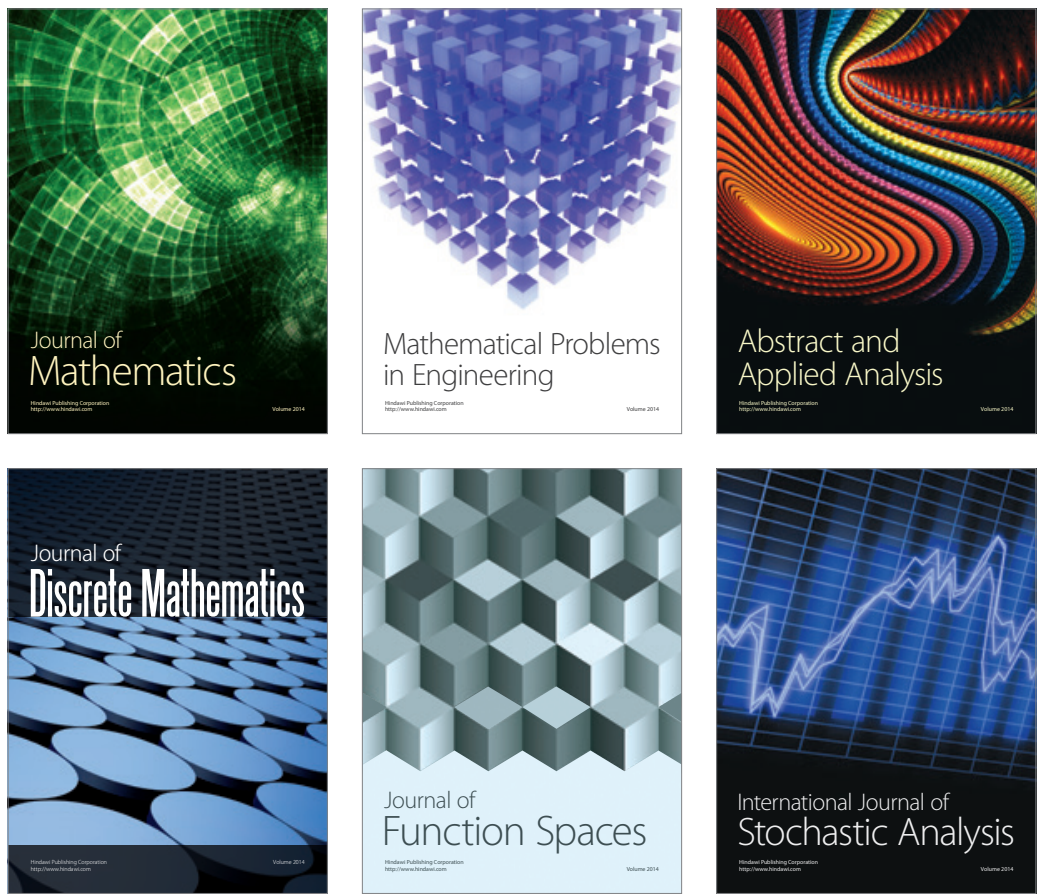

Journal of

Function Spaces

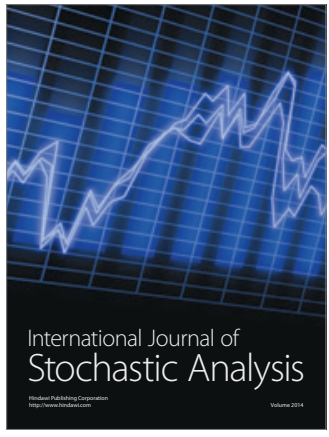

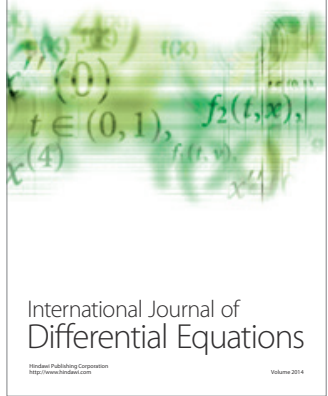
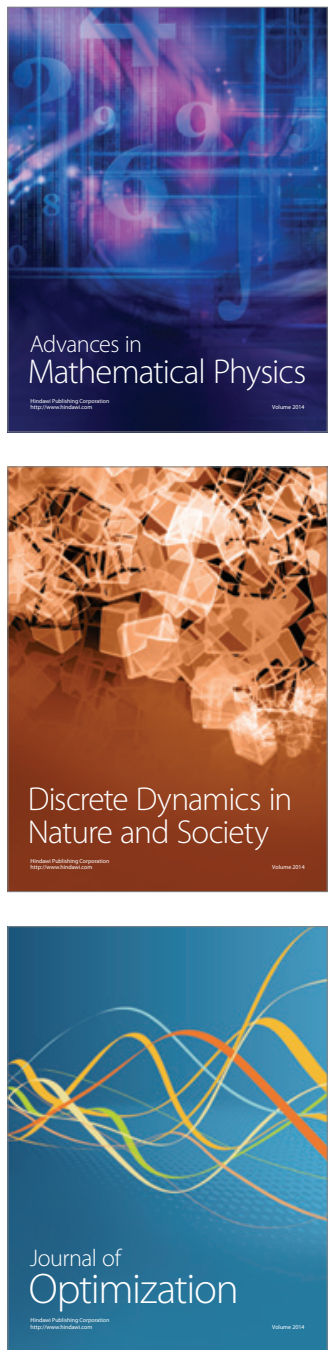